\title{
Coordinating a Supply Chain When Manufacturer Makes Cost Reduction Investment in Supplier
}

\author{
Shilei Huang, Hong Fu, and Yongkai Ma \\ School of Management and Economics, University of Electronic Science and Technology of China, Chengdu, Sichuan 611731, China \\ Correspondence should be addressed to Hong Fu; hongfu@uestc.edu.cn
}

Received 20 February 2016; Revised 4 May 2016; Accepted 11 May 2016

Academic Editor: Paolo Renna

Copyright (C) 2016 Shilei Huang et al. This is an open access article distributed under the Creative Commons Attribution License, which permits unrestricted use, distribution, and reproduction in any medium, provided the original work is properly cited.

\begin{abstract}
We consider a supply chain consisting of an upstream supplier and a downstream manufacturer, in which the supplier provides a component to the manufacturer, facing a price-sensitive and uncertain demand. The manufacturer makes cost reduction investment in the supplier to improve the supplier's production efficiency, which benefits the entire supply chain. We derive the optimal investment and operating decisions. Both the centralized and decentralized supply chains are studied. We show that the optimal investment and operating decisions in the decentralized setting may deviate from that in the centralized setting. To avoid the profit loss caused by such a deviation, we develop a coordination mechanism by introducing a combined policy of revenue-sharing policy and investment cost-sharing policy. We also show that the developed coordination mechanism can achieve Pareto improvement for the two players.
\end{abstract}

\section{Introduction}

Low production cost is a key factor in achieving competitive advantage and is significantly correlated with the firm's resources, such as technology, equipment, and other resources [1]. However, according to resource-based theory, valuable resources are scarce [2], and few firms have sufficient resources which can be used to reduce their production costs. To reduce the production cost, some upstream firms ask for help from their downstream firms. Intuitively, when the production cost of the upstream firm is reduced, the entire supply chain becomes more cost efficient, which will also benefit the downstream firm. Thus, the downstream firm has incentive to invest in the upstream firm's production cost reduction. As a matter of fact, in reality, many downstream firms have provided the needed resources for the upstream firms to reduce their production costs or enhance their production productivity. For example, in Japan, many downstream firms provide technology, equipment, and so forth, to upstream firms [3]. In Philippines, the downstream firms provide direct assistance, such as the training of management and staff, to upstream firms [4]. The resources provided by these downstream firms can lead to a production cost reduction for their upstream firms.
This paper is related to two streams of literature. The first stream focuses on the supply chain coordination. For example, Chakraborty and Chatterjee [5] coordinate a supply chain with deterministic yield and deterministic demand. Tang and Kouvelis [6] investigate coordination in a supply chain with uncertain yield and deterministic demand. Fu et al. [7] explore the coordination issue of a supply chain with deterministic yield and uncertain demand. Moreover, $\mathrm{Hu}$ et al. [8] and Fu et al. [9] study the coordination issue of a supply chain with uncertain yield and uncertain demand. In addition, Panda et al. [10] and Modak et al. [11] coordinate a three-layer supply chain consisting of a manufacturer, a distributer, and a retailer. Giri et al. [12] coordinate a three-layer supply chain with uncertain yield and uncertain demand. Although the above literature has investigated supply chain coordination from different perspectives, it does not consider cost reduction investment.

The second stream of literature concerns cost reduction investment. For example, In a one-to-one supply chain, $\mathrm{Ge}$ et al. [13] assume that both the upstream firm and the downstream firm can invest in their own production cost reduction and investigate the investment strategies for the two firms. Usta et al. [14] investigate the downstream firm's 
investment in its production cost reduction and show that the downstream firm's full commitment to invest in cost reduction enables the upstream firm to license technology and charge lower prices. In a one-to-many supply chain, Banerjee and Lin [15] investigate the upstream firm's investment in its production cost reduction and show that under the costsharing rules, there is a fundamental conflict between the incentives of the upstream firm and the downstream firms: the upstream firm prefers more downstream firms to participate in the cost-sharing rules, but the downstream firms may not be so. Banerjee and Lin [16] consider the problem that downstream firms invest in their own production cost reduction and show that by using fixed-price agreements under which the input price remains unchanged in response to downstream firms' investment strategies, higher investment levels will be chosen by downstream firms. In a many-to-one supply chain, Bernstein and Kök [17] investigate the problem that upstream firms invest in their own production cost reduction and show that target-price contracts lead to higher investment levels and profits if the rates are properly specified, as compared to the cost-contingent contracts. Bernstein et al. [18] explore the upstream firms' cost reduction investment problem by using cooperative game theory and show that the optimal investment level of the entire supply chain can by achieved when the most efficient upstream firm receives the incremental benefits of the cost reduction achieved by other upstream firms. See, Ishii [19], Gupta [20], and Li et al. [21] for more discussion on cost reduction investment in supply chains. Although existing studies have investigated the problem of cost reduction investment in supply chains from different perspectives, they do not explore the problem that a firm's production cost is reduced by its vertical supply chain partners.

In this paper, we consider that an upstream supplier sells a component to a downstream manufacturer. The manufacturer has a chance to invest in the supplier with specific resources, which can reduce the production cost of the supplier. We first explore the centralized supply chain, to provide a benchmark for the decentralized supply chain. Then, we study the decentralized supply chain. Finally, a mechanism is proposed to coordinate the decentralized supply chain. We consider both the cost reduction investment strategy and the operating decisions (i.e., pricing and production decisions). follows:

In summary, the key contributions of this paper are as

(1) We establish a model to investigate the problem of the cost reduction investment by the downstream manufacturer in the upstream supplier.

(2) We derive the optimal operating decisions of the supplier and the manufacturer, under any given cost reduction investment strategy of the manufacturer.

(3) We characterize the optimal cost reduction investment strategies for the manufacturer in both the centralized and decentralized supply chains.

(4) We develop a mechanism to coordinate the decentralized supply chain and show that the developed mechanism has sufficient flexibility to allocate the expected profit of the supply chain between the supplier and the manufacturer and thus can achieve Pareto improvement.

The rest of this paper is organized as follows. In Section 2, we present the model. In Section 3, we derive the optimal investment and operating decisions for the centralized supply chain. In Section 4, we characterize the equilibrium decisions of the two players in the decentralized supply chain. Section 5 propose a coordination mechanism for the decentralized supply chain. Section 6 concludes our work.

\section{The Model}

Consider a supply chain consisting of a single upstream supplier and a single downstream manufacturer. The manufacturer produces a product and sells it to a market with pricesensitive and uncertain demand. Without loss of generality, we suppose the demand function takes the form of

$$
D(p, \varepsilon)=y(p) \cdot \varepsilon,
$$

where $\varepsilon$ in (1) is the random factor which has a support on $[A, B], 0<A<B$, with a probability density function $f(x)$ and a cumulative distribution function $F(x)$. We suppose $\varepsilon$ satisfies the increasing generalized failure rate (IGFR) condition; that is, $x f(x) /(1-F(x))$ is increasing in $x[7,22]$. Moreover, $y(p)$ is the price-dependent part of demand and takes the form of

$$
y(p)=a p^{-b},
$$

where $a$ in (2) represents the market size and $b$ is the elasticity of demand with respect to the retail price, $b>1$ [23].

We assume that the manufacturer has a chance to make investment in the supplier. The manufacturer's investment may be technology, equipment, and other resources, which can lead to a cost reduction in the supplier's component. The value of the manufacturer's investment is denoted by $k$, which is constrained to be in $[0, K]$, where $K$ denotes the maximum value of resources that the manufacturer can invest. We assume that the production cost of the supplier is $c(k)$ and $c(k)$ is decreasing in $k$. If $k=0$, then $c(k)=c$, where $c$ represents the production cost of the supplier without the manufacture's investment. More specifically, we assume that $c(k)$ is linear in $k$ and takes the form of

$$
c(k)=c-\delta k,
$$

where $\delta$ in (3) represents the cost reduction efficiency of the manufacturer's investment. We require $\delta<c / K$, to guarantee that the production cost of the supplier is positive. We further assume that the manufacturing cost for the manufacturer is negligible, and one unit of product requires one unit of component from the supplier.

The decisions to be made by the supplier and the manufacturer fall in three stages. In the first stage, the manufacturer chooses its investment level $k$. In the second stage, the supplier sets a wholesale price $w$ for the component. In the last stage, the manufacturer decides an order quantity $q$ and the retail price $p$ for the product. 
Based upon the above description, the expected profit functions of the supplier and the manufacturer can be written as follows, respectively:

$$
\begin{aligned}
\Pi_{s}(w) & =w q-c(k) q, \\
\Pi_{m}(p, q \mid k) & =p E\{\min [q, D(p, \varepsilon)]\}-w q-k .
\end{aligned}
$$

In (5), the first term $p E\{\min [q, D(p, \varepsilon)]\}$ is the expected revenue of the manufacturer by selling the product to the market. The second term $w q$ represents the cost for the manufacturer to purchase the component. The last term $k$ corresponds to the investment cost for the manufacturer.

\section{Centralized Supply Chain}

In this section, we investigate the decision problem in the centralized setting, which serves as a benchmark for the decentralized setting. In the centralized supply chain, the supplier and the manufacturer make decisions to maximize their total expected profit, that is, the expected profit of the entire supply chain. From (4) and (5), we know that the expected profit of the entire supply chain is given by

$$
\begin{aligned}
\Pi_{c}(p, q \mid k) & =\Pi_{s}(w)+\Pi_{m}(p, q \mid k) \\
& =p E\{\min [q, D(p, \varepsilon)]\}-c(k) q-k .
\end{aligned}
$$

From (6), we can see that the wholesale price $w$ vanishes. This is not surprising, because $w q$ represents the internal transfer payment from the manufacturer to the supplier. Then, the decision problem is to choose values for $k, p$, and $q$, so that the profit $\Pi_{c}(p, q \mid k)$ is maximized.

Following Petruzzi and Dada [24], we define stocking factor $z=q / y(p)$. Then, the problem of choosing a retail price $p$ and an order quantity $q$ is equivalent to choosing a retail price $p$ and a stocking factor $z$. Substituting $z=q / y(p)$ into (6), then the expected profit of the entire supply chain can be rewritten as

$$
\begin{aligned}
\Pi_{c}(p, z \mid k) & =p y(p) E[\min (z, \varepsilon)]-c(k) z y(p)-k \\
& =y(p)[p h(z)-c(k) z]-k,
\end{aligned}
$$

where $h(z)=z-\int_{A}^{z} F(x) d x$.

We first present the following lemma.

Lemma 1. For any given investment level $k$, the optimal retail price $p_{c}^{*}(k)$ for the entire supply chain is given by

$$
p_{c}^{*}(k)=\frac{b z_{c}^{*} c(k)}{(b-1) h\left(z_{c}^{*}\right)},
$$

and the optimal stocking factor $z_{c}^{*}$ is uniquely determined by

$$
(b-1) h\left(z_{c}^{*}\right)=b\left[1-F\left(z_{c}^{*}\right)\right] z_{c}^{*} .
$$

Proof of Lemma 1. (i) Taking the first derivative of $\Pi_{c}(p, z \mid k)$ of (7) with respect to $p$ gives

$$
\frac{d \Pi_{c}(p, z \mid k)}{d p}=a p^{-b-1} T(p),
$$

where $T(p)=(1-b) p h(z)+b z c(k)$.
Obviously, $T(p)$ is linearly decreasing in $p$, and $\lim _{p \rightarrow 0} T(p)>0, \lim _{p \rightarrow+\infty} T(p)<0$. Thus, there exists a unique $\bar{p}$, such that $T(p)>0$ for $p<\bar{p}$, and $T(p)<$ 0 for $p>\bar{p}$. In conjunction with the fact that $a p^{-b-1}>$ 0 , we know that $d \Pi_{c}(p, z \mid k) / d p>0$ for $p<\bar{p}$ and $d \Pi_{c}(p, z \mid k) / d p<0$ for $p>\bar{p}$. Thus, $\Pi_{c}(p, z \mid k)$ is unimodal in $p$ and has a unique maximizer. Solving $d \Pi_{c}(p, z \mid k) / d p=a p^{-b-1} T(p)=0$ yields $\bar{p}=b z c(k) /$ $(b-1) h(z)$.

(ii) Substituting $p=\bar{p}$ into $\Pi_{c}(p, z \mid k)$ of (7), we get $\Pi_{c}(z \mid k)=z y(\bar{p}) c(k) /(b-1)-k$. Taking the first derivative of $\Pi_{c}(z \mid k)$ with respect to $z$ gives

$$
\frac{d \Pi_{c}(z \mid k)}{d z}=\frac{a c(k) \bar{p}^{-b}}{(b-1) h(z)} R(z),
$$

where $R(z)=(1-b) h(z)+b[1-F(z)] z$ and $d R(z) / d z=$ $[1-F(z)][1-b z f(z) /(1-F(z))]$.

Because $z f(z) /(1-F(z))$ is increasing in $z$ (IGFR condition), we can conclude that $R(z)$ is unimodal in $z$. Note that $a c(k) \bar{p}^{-b} /(b-1) h(z)>0$; we know that $\Pi_{c}(z \mid k)$ is unimodal in $z$ and has a unique maximizer. Solving $d \Pi_{c}(z \mid$ $k) / d z=0$ yields (9). Substituting $z=z_{c}^{*}$ of (9) into $\bar{p}=$ $b z c(k) /(b-1) h(z)$, we get (8). We thus complete the proof of Lemma 1.

Note that $z_{c}^{*}$ of (9) is not affected by the investment level $k$ and $c(k)$ decreases as the investment level $k$ increases. Then, we can see from Lemma 1 that the retail price $p_{c}^{*}(k)$ decreases as the investment level $k$ increases. Moreover, because $z=$ $q / y(p)$, then Lemma 1 indicates that the corresponding order quantity $q_{c}^{*}(k)=z_{c}^{*} y\left(p_{c}^{*}(k)\right)$ increases as the investment level $k$ increases. Substituting $c(k)$ of $(3), p_{c}^{*}(k)$ of $(8)$, and $z_{c}^{*}$ of (9) into (7), we can show that the expected profit of the supply chain can be expressed as

$$
\Pi_{c}(k)=\frac{a(b-1)^{b-1} h^{b}\left(z_{c}^{*}\right)}{b^{b}\left(z_{c}^{*}\right)^{b-1}(c-\delta k)^{b-1}}-k .
$$

Now, we address the optimal investment strategy for the entire supply chain. We first denote

$$
\delta_{1}=\frac{c}{K}-\frac{1}{K}\left(\frac{M c^{b-1}}{K c^{b-1}+M}\right)^{1 /(b-1)}
$$

where $M=a(b-1)^{b-1} h^{b}\left(z_{c}^{*}\right) / b^{b}\left(z_{c}^{*}\right)^{b-1}$. Then, we can present the following theorem.

Theorem 2. (i) If $\delta<\delta_{1}$, then the optimal investment strategy for the entire supply chain is $k_{c}^{*}=0$; (ii) if $\delta>\delta_{1}$, then the optimal investment strategy for the entire supply chain is $k_{c}^{*}=$ K.

Proof of Theorem 2. Taking the first derivative of $\Pi_{c}(k)$ of (12) with respect to $k$ gives

$$
\frac{d \Pi_{c}(k)}{d k}=\frac{\delta M(b-1)}{(c-\delta k)^{b}}-1 .
$$


Obviously, $d \Pi_{c}(k) / d k$ is increasing in $\delta$. Thus, we have $d^{2} \Pi_{c}(k) / d k^{2}>0$. That is, $\Pi_{c}(k)$ is strictly convex in $\delta$ and has the unique maximizer $k=0$ or $k=M$. Solving $\Pi_{c}(0)>$ $\Pi_{c}(M)$ yields Theorem 2(i); solving $\Pi_{c}(0)<\Pi_{c}(M)$ yields Theorem 2(ii). We thus complete the proof of Theorem 2.

From Theorem 2, we know that the optimal investment strategy for the entire supply chain is dependent on cost reduction efficiency $\delta$ and follows an extreme policy: investment strategy is either zero or maximum. Specifically, when cost reduction efficiency $\delta$ is relatively low, the optimal investment strategy for the entire supply chain is $k_{c}^{*}=$ 0 ; when cost reduction efficiency $\delta$ is relatively high, the optimal investment strategy for the entire supply chain is $k_{c}^{*}=K$.

Substituting $p=p_{c}^{*}(k), z=z_{c}^{*}$, and $k=k_{c}^{*}$ into (7), we can obtain the optimal expected profit of the centralized supply chain, denoted by $\Pi_{c}^{*}$. Next, we consider the decentralized supply chain.

\section{Decentralized Supply Chain}

In the decentralized supply chain, the supplier and the manufacturer are independent decision makers and make decisions to maximize their own expected profits. We analyze the decision problem, using a three-stage approach.

4.1. The Third Stage. Given the manufacturer's investment level $k$, and the supplier's wholesale price $w$, the manufacturer chooses an order quantity $q$ and a retail price $p$ for the product to maximize its expected profit.

From (5), we have the following lemma.

Lemma 3. For any given investment level $k$ and wholesale price $w$, the optimal retail price $p^{*}(w, k)$ and the order quantity $q^{*}(w, k)$ for the manufacturer are given by

$$
\begin{aligned}
p^{*}(w, k) & =\frac{b z_{c}^{*} w}{(b-1) h\left(z_{c}^{*}\right)}, \\
q^{*}(w, k) & =\frac{a(b-1)^{b} h^{b}\left(z_{c}^{*}\right)}{b^{b}\left(z_{c}^{*}\right)^{b-1} w^{b}} .
\end{aligned}
$$

Proof of Lemma 3. First, similar to the proof of Lemma 1, it is easy to show that, in decentralized supply chain, for any given investment level $k$ and wholesale price $w$, the optimal retail price is $p^{*}(w, k)$ of (15). Second, combining $p=p^{*}(w, k)$ of (15) and $z=q / y(p)$, we then have that the optimal order quantity is $q^{*}(w, k)$ of $(16)$. We thus complete the proof of Lemma 3.

Lemma 3 gives the manufacturer's best response retail price $p^{*}(w, k)$ and order quantity $q^{*}(w, k)$ to the investment level $k$ and wholesale price $w$. From Lemma 3, we can see that, for any given investment level $k$, the manufacturer's best response retail price $p^{*}(w, k)$ increases as the wholesale price $w$ increases, and the best response order quantity $q^{*}(w, k)$ decreases as the wholesale price $w$ increases. Lemma 3 does not provide an answer to how the investment level $k$ affects the manufacturer's best response retail price $p^{*}(w, k)$ and order quantity $q^{*}(w, k)$. As a matter of fact, the investment level $k$ affects the manufacturer's choices of $p^{*}(w, k)$ and $q^{*}(w, k)$ via wholesale price $w$, which will be verified in Lemma 4.

4.2. The Second Stage. Knowing that the manufacturer chooses the retail price and the order quantity according to (15) and (16), respectively, the supplier chooses a wholesale price $w$ to maximize its expected profit. By substituting $q^{*}(w, k)$ of (16) into (4), we can obtain the supplier's expected profit function as follows:

$$
\Pi_{s}(w)=[w-c(k)] \frac{a(b-1)^{b} h^{b}\left(z_{c}^{*}\right)}{b^{b}\left(z_{c}^{*}\right)^{b-1} w^{b}},
$$

From (17), we have the following lemma.

Lemma 4. For any given investment level $k$, the optimal wholesale price $w^{*}(k)$ for the supplier is given by

$$
w^{*}(k)=\frac{b c(k)}{b-1} .
$$

Proof of Lemma 4. Taking the first derivative of $\Pi_{s}(w)$ of (17) with respect to $w$ gives

$$
\frac{d \Pi_{s}(w)}{d w}=\frac{a(b-1)^{b} h^{b}\left(z_{c}^{*}\right)}{b^{b}\left(z_{c}^{*}\right)^{b-1} w^{b+1}} V(w),
$$

where $V(w)=(1-b) w+b c(k)$.

Obviously, $V(w)$ is linearly decreasing in $w$, and $\lim _{w \rightarrow 0} V(w)>0, \lim _{w \rightarrow+\infty} V(w)<0$. Thus, there exists a unique $\bar{w}$, such that $V(w)>0$ for $w<\bar{w}$ and $V(w)<$ 0 for $w>\bar{w}$. In conjunction with the fact that $a(b-1)^{b} h^{b}\left(z_{c}^{*}\right) /$ $b^{b}\left(z_{c}^{*}\right)^{b-1} w^{b+1}>0$, we know that $d \Pi_{s}(w) / d w>0$ for $w<\bar{w}$ and $d \Pi_{s}(w) / d w<0$ for $w>\bar{w}$. Thus, $\Pi_{s}(w)$ is unimodal in $w$ and has a unique maximizer. Solving $d \Pi_{s}(w) / d w=0$ yields (18). We thus complete the proof of Lemma 4.

Lemma 4 characterizes the supplier's best response wholesale price $w^{*}(k)$ to the investment level $k$ and states that the best response wholesale price $w^{*}(k)$ decreases as the investment level $k$ increases. This is not surprising, because an increase in $k$ leads to a lower production cost $c(k)$. Then, the supplier with a lower production cost will charge a lower wholesale price to the manufacturer. Second, in conjunction with the discussion following Lemma 3, we can conclude that the investment level $k$ will affect the manufacturer's choices of $q^{*}(w, k)$ and $p^{*}(w, k)$ via $w^{*}(k)$.

4.3. The First Stage. Knowing the operating decisions (i.e., pricing and production decisions) response to the investment decision, according to (15), (16), and (18), the manufacturer chooses its investment level $k$. Substituting $c(k)$ of (3), $p^{*}(w, k)$ of (15), $q^{*}(w, k)$ of (16), and $w^{*}(k)$ of (18) into (4) and (5), respectively, the expected profit functions of the 
suppler and the manufacturer can be rewritten as follows, respectively:

$$
\begin{aligned}
\Pi_{s}(k) & =\frac{a(b-1)^{2 b-1} h^{b}\left(z_{c}^{*}\right)}{b^{2 b}\left(z_{c}^{*}\right)^{b-1}(c-\delta k)^{b-1}}, \\
\Pi_{m}(k) & =\frac{a(b-1)^{2 b-2} h^{b}\left(z_{c}^{*}\right)}{b^{2 b-1}\left(z_{c}^{*}\right)^{b-1}(c-\delta k)^{b-1}}-k .
\end{aligned}
$$

The following corollary can be made according to (20) and (21).

Corollary 5. The supplier can always benefit from the manufacturer's investment, while the investment may cause detriment to the manufacturer's profit.

Proof of Corollary 5. Corollary 5 can be obtained directly from (20) and (21). Thus, it is omitted here.

When the manufacturer invests in the supplier, the production cost of the component is reduced, and the supply chain becomes more cost efficient, which benefits both the supplier and the manufacturer. From (20) and (21), we can calculate that the benefit caused by the cost reduction for the supplier is

$$
\begin{aligned}
\Delta \Pi_{s} & =\Pi_{s}(k)-\Pi_{s}(0) \\
& =\frac{a(b-1)^{2 b-1} h^{b}\left(z_{c}^{*}\right)}{b^{2 b}\left(z_{c}^{*}\right)^{b-1}}\left[\frac{1}{(c-\delta k)^{b-1}}-\frac{1}{c^{b-1}}\right]
\end{aligned}
$$

and the benefit caused by the cost reduction for the manufacturer is

$$
\begin{aligned}
\Delta \Pi_{m} & =\left[\Pi_{m}(k)+k\right]-\left[\Pi_{m}(0)+0\right] \\
& =\frac{a(b-1)^{2 b-2} h^{b}\left(z_{c}^{*}\right)}{b^{2 b-1}\left(z_{c}^{*}\right)^{b-1}}\left[\frac{1}{(c-\delta k)^{b-1}}-\frac{1}{c^{b-1}}\right] .
\end{aligned}
$$

Thus, the supplier is willing to be invested. However, the manufacturer may not invest, because manufacturer should weigh up the investment cost $k$ and the benefit caused by the cost reduction $\Delta \Pi_{m}$. Specially, when $k<\Delta \Pi_{m}$, the manufacturer should invest; otherwise, it is not necessary for the manufacturer to invest.

Next, we address the manufacturer's investment strategy in decentralized supply chain. Denote

$$
\delta_{2}=\frac{c}{K}-\frac{1}{K}\left(\frac{N c^{b-1}}{K c^{b-1}+N}\right)^{1 /(b-1)}
$$

where $N=a(b-1)^{2 b-2} h^{b}\left(z_{c}^{*}\right) / b^{2 b-1}\left(z_{c}^{*}\right)^{b-1}$. We can establish the following theorem according to (21) and (24).

Theorem 6. (i) If $\delta<\delta_{2}$, then the optimal investment strategy for the manufacturer is $k^{*}=0$; (ii) if $\delta>\delta_{2}$, then the optimal investment strategy for the manufacturer is $k^{*}=K$.
Proof of Theorem 6. This proof is analog to the proof of Theorem 2. Thus, it is omitted here.

Substituting $k=k^{*}$ into (20) and (21), we can obtain the optimal expected profits of the supplier and the manufacturer, denoted by $\Pi_{s}^{*}$ and $\Pi_{m}^{*}$, respectively.

Similar to the optimal investment strategy in the centralized supply chain, the optimal investment strategy in the decentralized supply chain also follows the extreme policy. That is, investment level is either zero or maximum. In the rest of this subsection, we compare the results obtained in centralized and decentralized supply chains, to get more insights.

From (8) and (9), (15) and (16), and (18), we have the following corollary.

Corollary 7. For any given investment level $k$ (i) the optimal retail price in centralized supply chain is lower than that in the decentralized supply chain; that is, $p_{c}^{*}(k)<p^{*}\left(w^{*}(k), k\right)$; (ii) the optimal order quantity in centralized supply chain is higher than that in the decentralized supply chain; that is, $q_{c}^{*}(k)=$ $z_{c}^{*} y\left(p_{c}^{*}(k)\right)>q^{*}\left(w^{*}(k), k\right)$.

Proof of Corollary 7. (i) First, it follows from (8), (15), and (18) that

$$
p^{*}\left(w^{*}(k), k\right)-p_{c}^{*}(k)=\frac{b z_{c}^{*} c(k)}{(b-1)^{2} h\left(z_{c}^{*}\right)}>0 .
$$

(ii) Second, it follows from (8) and (9), (16), and (18) that

$$
\begin{aligned}
q^{*} & \left(w^{*}(k), k\right)-q_{c}^{*}(k) \\
& =q^{*}\left(w^{*}(k), k\right)-z_{c}^{*} y\left(p_{c}^{*}(k)\right) \\
& =\frac{a(b-1)^{b} h^{b}\left(z_{c}^{*}\right)}{b^{b}\left(z_{c}^{*}\right)^{b-1} c^{b}(k)}\left[\left(1-\frac{1}{b}\right)-1\right]<0 .
\end{aligned}
$$

Combining (i) and (ii), we complete the proof of Corollary 7.

When the investment level is given, the expected profit of the supply chain is determined by the retail price and the order quantity. Corollary 7 shows that the optimal operating decisions in the decentralized setting, deviate from that in the centralized setting. Such deviation will cause the profit loss for the decentralized supply chain. This is because the well known double marginalization [25] exists between the supplier and the manufacturer in the decentralized supply chain.

Note from (13) and (24) that $N / M=(b-1)^{b-1} / b^{b-1}<$ 1. Then, we have $\delta_{1}<\delta_{2}$. This, together with Theorem 2 and Theorem 6 , allows us to obtain the following important corollary.

Corollary 8. (i) If $\delta<\delta_{1}$, the optimal investment strategies in both the centralized and decentralized supply chains are zero; that is, $k_{c}^{*}=0$ and $k^{*}=0$; (ii) if $\delta>\delta_{2}$, the optimal investment strategies in both the centralized and decentralized 
supply chains are maximum; that is, $k_{c}^{*}=K$ and $k^{*}=K$; (iii) if $\delta_{1}<\delta<\delta_{2}$, the optimal investment strategy in the centralized supply chain is maximum, while the optimal investment strategy in the decentralized supply chain is zero; that is, $k_{c}^{*}=M$ and $k^{*}=0$.

Although the optimal investment strategies in the centralized and decentralized settings are both either zero or maximum, the specific optimal investment strategies may be different. Specifically, when cost reduction efficiency is low to some degree, that is, $\delta<\delta_{1}$, the optimal investment strategies in two settings are both zero; when cost reduction efficiency is high to some degree, that is, $\delta>\delta_{2}$, the optimal investment strategies in two settings are both maximum; otherwise, $\delta_{1}<\delta<\delta_{2}$, the optimal investment strategy in the decentralized setting is zero, while the optimal investment strategy in the centralized setting is maximum. Corollary 8 states that when cost reduction efficiency is lower than $\delta_{1}$, the benefit from cost reduction can not outweigh the investment cost in the two settings, and, hence, it is no necessary to invest; when cost reduction efficiency is higher than $\delta_{2}$, the benefit from cost reduction outweighs the investment cost in the two settings, and, thus, it should invest. More importantly, Corollary 8 reveals that when cost reduction efficiency is in the range $\left(\delta_{1}, \delta_{2}\right)$, the entire supply chain can benefit from the investment, while the manufacturer can not benefit from the investment. This is because the benefit from the supplier's cost reduction caused by the manufacturer's investment is divided between the supplier and the manufacturer. That is, although the investment cost is undertaken by the manufacturer independently, the benefit from the investment can not be wholly obtained by the manufacturer. Such a result may affect the manufacturer's investment enthusiasm. Finally, we point out that when cost reduction efficiency is in the range $\left(\delta_{1}, \delta_{2}\right)$, the optimal investment strategy in the decentralized setting deviates from that in the centralized setting and causes the profit loss for the entire supply chain.

\section{Coordination Mechanism}

In Section 4, we have shown that the optimal investment and operating decisions in the decentralized setting may deviate from that in the centralized setting. In this section, we propose a mechanism to coordinate the decentralized supply chain, and then the optimal investment and operating decisions in the centralized setting will be chosen in the decentralized setting. In addition to the wholesale price policy, the proposed mechanism contains other two policies: a revenue-sharing policy and an investment cost-sharing policy. Specifically

(a) the revenue-sharing policy suggests that the supplier shares $\phi$ percentage of the manufacturer's sales revenue, $0<\phi<1$;

(b) the investment cost-sharing policy suggests that the supplier shares $\psi$ percentage of the manufacturer's investment cost, $0<\psi<1$.
Under the proposed mechanism, the expected profit functions of the supplier and the manufacturer can be expressed as follows, respectively:

$$
\begin{aligned}
\pi_{s}(w)= & w q-c(k) q+\phi p E\{\min [q, D(p, \varepsilon)]\} \\
& -\psi k, \\
\pi_{m}(p, q \mid k)= & p E\{\min [q, D(p, \varepsilon)]\}-w q-k \\
& -\phi p E\{\min [q, D(p, \varepsilon)]\}+\psi k .
\end{aligned}
$$

We are now ready to establish the following important theorem.

Theorem 9. For any given $0<\phi<1$, let $\psi=\phi$, and $w=$ $(1-\phi) c(k)$. Then, the decentralized supply chain is coordinated; that is, $p=p_{c}^{*}(k), q=q_{c}^{*}(k)$, and $k=k_{c}^{*}$.

Proof of Theorem 9. Substituting $\psi=\phi$ and $w=(1-\phi) c(k)$ into (27) and (28), respectively, the expected profit functions of the supplier and the manufacturer can be rewritten as follows, respectively:

$$
\begin{aligned}
\pi_{s} & =\phi\{p E\{\min [q, D(p, \varepsilon)]\}-c(k) q-k\} \\
& =\phi \Pi_{c}(p, q \mid k), \\
\pi_{m} & =(1-\phi)\{p E\{\min [q, D(p, \varepsilon)]\}-c(k) q-k\} \\
& =(1-\phi) \Pi_{c}(p, q \mid k) .
\end{aligned}
$$

Clearly, given $0<\phi<1$, both the supplier and the manufacturer prefer $p=p_{c}^{*}(k), q=q_{c}^{*}(k)$, and $k=k_{c}^{*}$, which maximize the expected profits of the entire supply chain, supplier, and the manufacturer. We thus complete the proof of Theorem 9.

Theorem 9 implies that the decentralized supply chain can be coordinated by the proposed mechanism with appropriate parameters. As a matter of fact, in reality, an acceptable coordination mechanism should guarantee that each player's expected profit is not worse off and at least one player is strictly better off, that is, Pareto improvement $[26,27]$. In the rest of this section, we will address that the expected profit of the coordinated supply chain under the proposed mechanism can be arbitrarily divided between the supplier and the manufacturer, and thus both the supplier and the manufacturer can benefit from the coordination.

Substituting $p=p_{c}^{*}(k), q=q_{c}^{*}(k), k=k_{c}^{*}, \psi=\phi$, and $w=(1-\phi) c(k)$ into (27) and (28), respectively, we know that the expected profits of the supplier and the manufacturer under the proposed mechanism, are as follows, respectively:

$$
\begin{aligned}
\pi_{s} & =\phi \Pi_{c}^{*}, \\
\pi_{m} & =(1-\phi) \Pi_{c}^{*},
\end{aligned}
$$

where $\Pi_{c}^{*}$ denotes the optimal expected profit of the centralized supply chain.

From (30), we can see that the expected profit of the supplier $\pi_{s}$ increases in the revenue share $\phi$, and the expected 
profit of the manufacturer $\pi_{m}$ decreases in the revenue share $\phi$. Moreover, if $\phi \rightarrow 0$, then $\pi_{s} \rightarrow 0$ and $\pi_{m} \rightarrow \Pi_{c}^{*}$; if $\phi \rightarrow 1$, then $\pi_{s} \rightarrow \Pi_{c}^{*}$ and $\pi_{m} \rightarrow 0$. Accordingly, the expected profit of the supply chain under coordination can be divided between the supplier and the manufacturer arbitrarily; the parameter $\left\{w_{i}\right\}$ which enables the supplier and the manufacturer to obtain higher expected profits must exist. This is summarized as the following corollary.

Corollary 10. If $\Pi_{s}^{*} / \Pi_{c}^{*}<\phi<1-\Pi_{m}^{*} / \Pi_{c}^{*}$, then both the supplier and the manufacturer can benefit from the proposed mechanism; that is, $\pi_{s}>\Pi_{s}^{*}$ and $\pi_{m}>\Pi_{m}^{*}$, where $\Pi_{s}^{*}$ and $\Pi_{m}^{*}$ are the expected profits of the supplier and the manufacturer without coordination mechanism, respectively.

Proof of Corollary 10. (i) Solving $\pi_{s}=\phi \Pi_{c}^{*}>\Pi_{s}^{*}$ yields $\phi>\Pi_{s}^{*} / \Pi_{c}^{*}$; (ii) solving $\pi_{m}=(1-\phi) \Pi_{c}^{*}>\Pi_{m}^{*}$ yields $\phi<1-\Pi_{m}^{*} / \Pi_{c}^{*}$. Combining (i) and (ii), we complete the proof of Corollary 10.

Corollary 10 provides the feasible set of the revenue share such that the supplier and the manufacturer are both willing to participate in the coordination. The feasible set reveals the following managerial insights. Although the supplier has an incentive to raise the revenue share $\phi$, the manufacturer's participation condition $\pi_{m}>\Pi_{m}^{*}$ implies an upper bound of the revenue share $\phi$; that is, $\phi<1-\Pi_{m}^{*} / \Pi_{c}^{*}$. Similarly, the motivation of the manufacturer to decrease the revenue share $\phi$ is constrained by the supplier's participation condition $\pi_{s}>$ $\Pi_{s}^{*}$. In general, the actual value of $\phi$ depends on the two players' relative bargaining power.

\section{Conclusion}

This paper is motivated by the practice that the production cost of the upstream firm can be reduced by the downstream firm's investment in the decentralized supply chain. Although there has been some studies on cost reduction investment in decentralized supply chains, they assume that the production cost of a supply chain member is reduced by the investment of itself and do not consider the problem of the cost reduction investment by the supply chain member in its vertical partners. This paper investigates cost reduction investment by the downstream manufacturer in the upstream supplier. Both the centralized and the decentralized supply chains are investigated, and a mechanism is developed to coordinate the decentralized supply chain. The main results of this paper are summarized as follows.

(1) In the decentralized supply chain, the supplier can always benefit from the manufacturer's cost reduction investment, while the manufacturer may or may not benefit from the investment.

(2) The optimal investment levels in both the centralized and decentralized supply chains follow an extreme policy. That is, the optimal investment level in each setting is either zero or maximum.

(3) The optimal order quantity in the decentralized supply chain is less than that in the centralized supply chain; the optimal retail price in the decentralized supply chain is higher than that in the centralized supply chain; the optimal investment level in the decentralized supply chain may be lower than that in the centralized supply chain.

(4) The expected profit of the decentralized supply chain is less than that of the centralized supply chain. The mechanism consisting of a wholesale price policy, a revenue-sharing policy, and an investment costsharing policy can be used to coordinate the supply chain by choosing mechanism parameters appropriately.

There exist many potential directions for future research. An interesting issue is to consider the manufacturer's cost reduction investment in suppliers in a many-to-one supply chain, in which the manufacturer chooses a set of the investment strategies. It is also interesting to consider the upstream supplier's cost reduction investment in the downstream manufacturer.

\section{Competing Interests}

The authors declare that they have no competing interests.

\section{Acknowledgments}

This paper is supported by the National Natural Science Foundation of China (no. 71531003).

\section{References}

[1] J. B. Jin, C. S. Leem, and C. H. Lee, "Research issues and trends in industrial productivity over 44 years," International Journal of Production Research, vol. 54, no. 5, pp. 1273-1284, 2016.

[2] J. J. Chrisman and W. E. McMullan, "A preliminary assessment of outsider assistance as a knowledge resource: the longer-term impact of new venture counseling," Entrepreneurship: Theory and Practice, vol. 24, no. 3, p. 37, 2000.

[3] J. H. Dyer and W. G. Ouchi, "Japanese-style partnerships: giving companies a competitive edge," Sloan Management Review, vol. 35, no. 1, pp. 51-63, 1993.

[4] H. Hill, "Subcontracting, technological diffusion, and the development of small enterprise in Philippine manufacturing," Journal of Developing Areas, vol. 19, no. 2, pp. 245-262, 1985.

[5] A. Chakraborty and A. K. Chatterjee, "A surcharge pricing scheme for supply chain coordination under JIT environment," European Journal of Operational Research, vol. 253, no. 1, pp. 1424, 2016.

[6] S. Y. Tang and P. Kouvelis, "Pay-back-revenue-sharing contract in coordinating supply chains with random yield," Production and Operations Management, vol. 23, no. 12, pp. 2089-2102, 2014.

[7] H. Fu, Y. Ma, and X. Tang, "A note on revenue-sharing vs. wholesale-price contracts in assembly systems with random demand," in Proceedings of the 12th International Conference on Service Systems and Service Management, 2015.

[8] F. Hu, C.-C. Lim, Z. Lu, and X. Sun, "Coordination in a singleretailer two-supplier supply chain under random demand and 
random supply with disruption," Discrete Dynamics in Nature and Society, vol. 2013, Article ID 484062, 12 pages, 2013.

[9] H. Fu, Y. Ma, D. Ni, and X. Cai, "Coordinating a decentralizedhybrid push-pull assembly system with unreliable supply and uncertain demand," Annals of Operations Research, 2015.

[10] S. Panda, N. M. Modak, M. Basu, and S. K. Goyal, "Channel coordination and profit distribution in a social responsible three-layer supply chain," International Journal of Production Economics, vol. 168, pp. 224-233, 2015.

[11] N. M. Modak, S. Panda, and S. S. Sana, "Three-echelon supply chain coordination considering duopolistic retailers with perfect quality products," International Journal of Production Economics, 2015.

[12] B. C. Giri, S. Bardhan, and T. Maiti, "Coordinating a threelayer supply chain with uncertain demand and random yield," International Journal of Production Research, vol. 54, no. 8, pp. 2499-2518, 2016.

[13] Z. Ge, Q. Hu, and Y. Xia, "Firms' R\&D cooperation behavior in a supply Chain," Production and Operations Management, vol. 23, no. 4, pp. 599-609, 2014.

[14] M. Usta, F. Erhun, and W. H. Hausman, "Supply licensing when the manufacturer strategically commits to invest in R\&D," Naval Research Logistics, vol. 61, no. 4, pp. 341-350, 2014.

[15] S. Banerjee and P. Lin, "Vertical research joint ventures," International Journal of Industrial Organization, vol. 19, no. 1-2, pp. 285-302, 2001.

[16] S. Banerjee and P. Lin, "Downstream R\&D, raising rivals' costs, and input price contracts," International Journal of Industrial Organization, vol. 21, no. 1, pp. 79-96, 2003.

[17] F. Bernstein and A. G. Kök, "Dynamic cost reduction through process improvement in assembly networks," Management Science, vol. 55, no. 4, pp. 552-567, 2009.

[18] F. Bernstein, A. Gürhan Kök, and A. Meca, "Cooperation in assembly systems: the role of knowledge sharing networks," European Journal of Operational Research, vol. 240, no. 1, pp. 160-171, 2015.

[19] A. Ishii, "Cooperative R\&D between vertically related firms with spillovers," International Journal of Industrial Organization, vol. 22, no. 8-9, pp. 1213-1235, 2004.

[20] S. Gupta, "Research note-channel structure with knowledge spillovers," Marketing Science, vol. 27, no. 2, pp. 247-261, 2008.

[21] H. Li, Y. Wang, R. Yin, T. J. Kull, and T. Y. Choi, "Target pricing: demand-side versus supply-side approaches," International Journal of Production Economics, vol. 136, no. 1, pp. 172-184, 2012.

[22] K. Rosling, "Inventory cost rate functions with nonlinear shortage costs," Operations Research, vol. 50, no. 6, pp. 1007-1017, 2002.

[23] Y. Wang, L. Jiang, and Z.-J. Shen, “Channel performance under consignment contract with revenue sharing," Management Science, vol. 50, no. 1, pp. 34-47, 2004.

[24] N. C. Petruzzi and M. Dada, "Pricing and the newsvendor problem: a review with extensions," Operations Research, vol. 47, no. 2, pp. 183-194, 1999.

[25] J. J. Spengler, "Vertical integration and antitrust policy," Journal of Political Economy, vol. 58, no. 4, pp. 347-352, 1950.

[26] G. P. Cachon, "Supply chain coordination with contracts," in Handbooks in Operations Research and Management Science, North Holland Press, 2003.
[27] L. Ren, Y. He, and H. Song, "Price and service competition of dual-channel supply chain with consumer returns," Discrete Dynamics in Nature and Society, vol. 2014, Article ID 565603, 10 pages, 2014. 


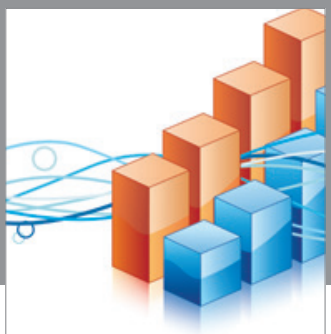

Advances in

Operations Research

vatem alat4

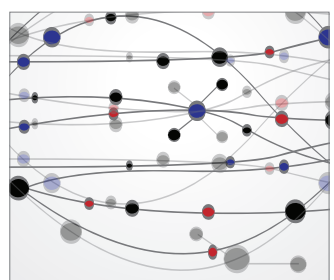

\section{The Scientific} World Journal
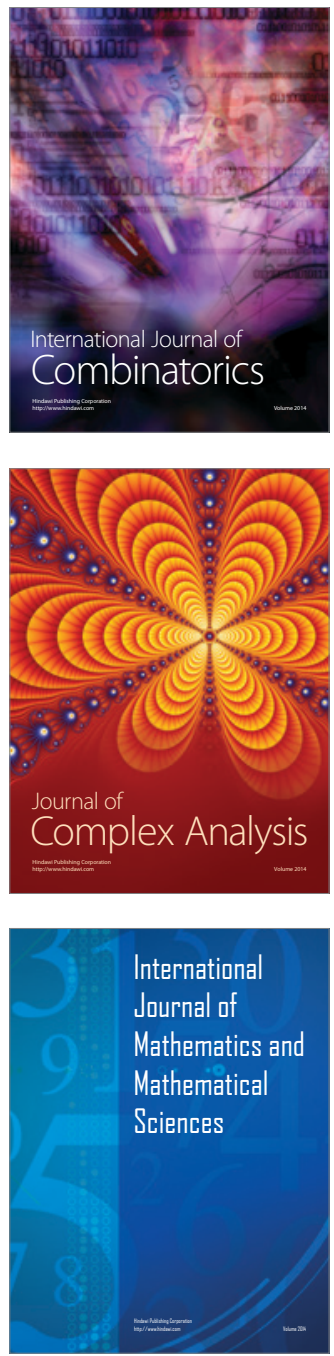
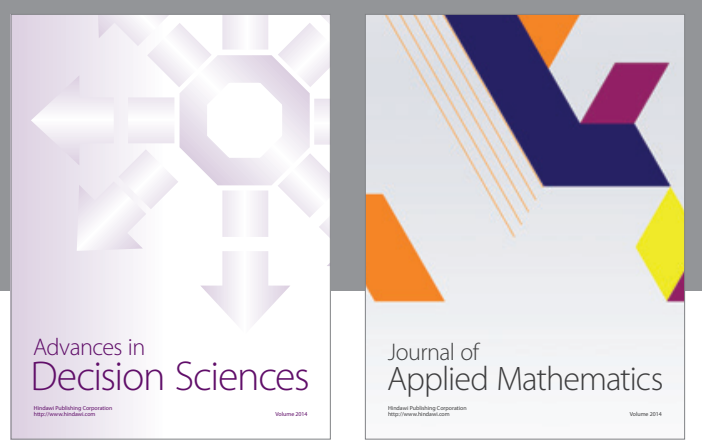

Algebra

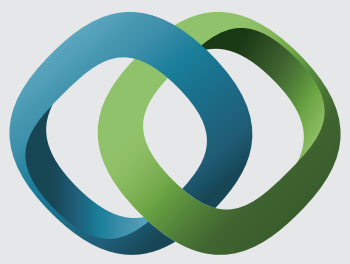

\section{Hindawi}

Submit your manuscripts at

http://www.hindawi.com
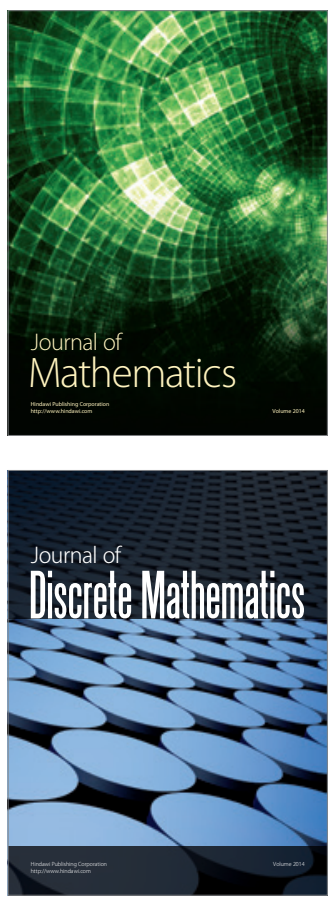

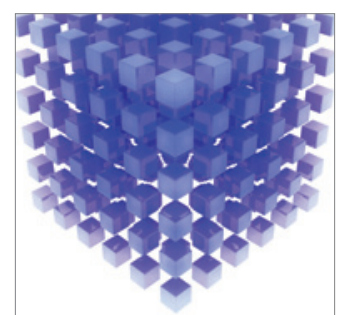

Mathematical Problems in Engineering
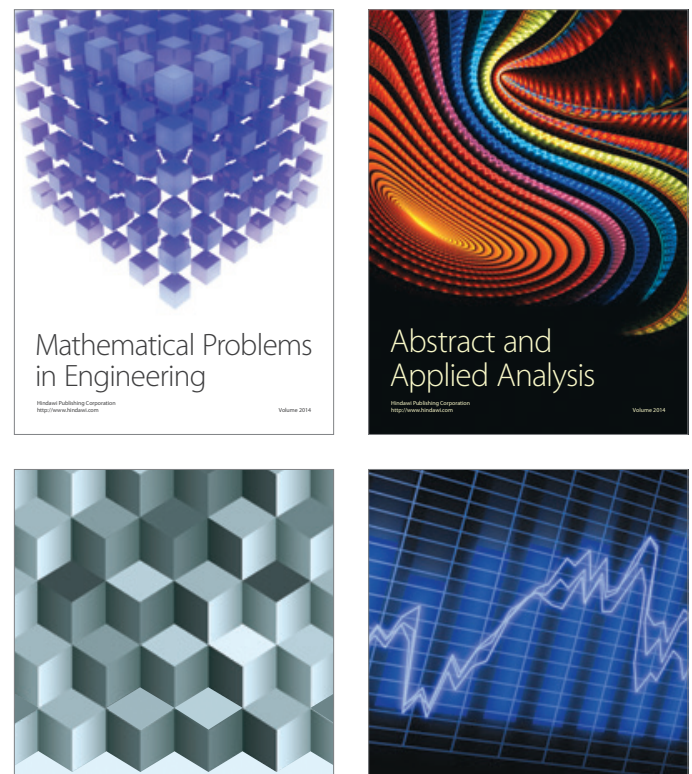

Journal of

Function Spaces

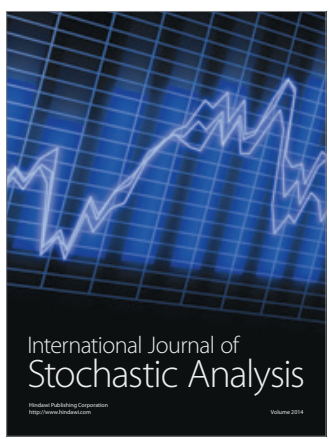

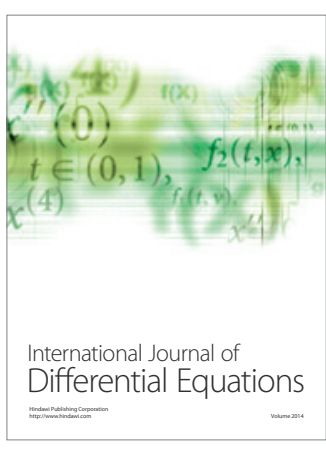
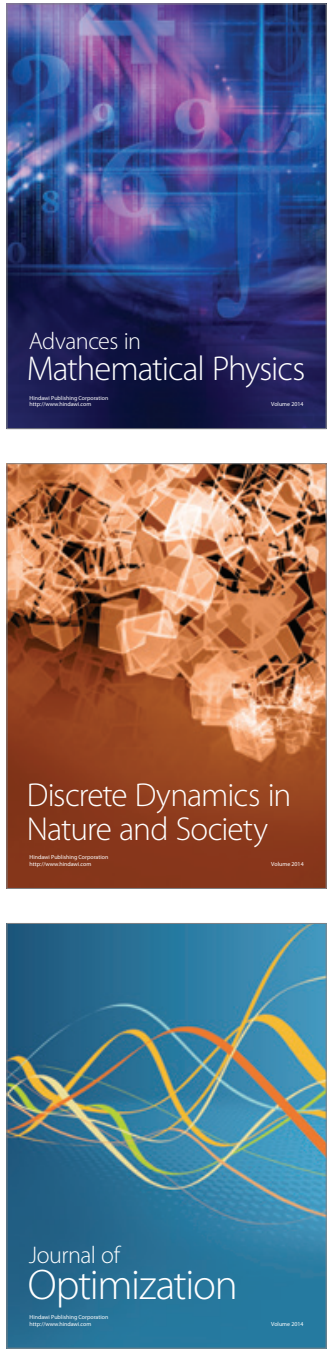